\title{
Dynamics of foot-and-mouth disease virus replication in cells at different phases of the cell-division cycle
}

\author{
Claudia DOEL ${ }^{1}$, Zhidong ZHANG $(\bowtie)^{1,2}$, Lise MAZELET ${ }^{1}$, Ryan WATERS ${ }^{1}$, John BASHIRUDDIN ${ }^{1}$ \\ 1 Pirbright Institute, Ash Road, Pirbright, Woking, Surrey, GU24 0NF, United Kingdom \\ 2 State Key Laboratory of Veterinary Etiological Biology, Lanzhou Veterinary Research Institute, Lanzhou730046, China
}

\begin{abstract}
Foot-and-mouth-disease virus (FMDV) replicates in epithelial cells. The restriction of FMDV RNA to the basal cell layer of epithelia suggests a possible link between FMDV replication in vivo and the cell status. This paper describes in vitro studies in which FMDV infection was investigated in cells that were held at various cell division phases using cell cycle inhibitors. The results suggest that when cells were arrested at the $G_{1}$ or $G_{1} / S$ phase, high levels of viral RNA were detected by quantitative real-time reverse transcription PCR and viral protein synthesis was observed by specific labeling techniques. In contrast, when cells were arrested at the $\mathrm{G}_{2} / \mathrm{M}$ phase, reduced or no viral RNA synthesis was detected.
\end{abstract}

Keywords foot-and-mouth disease virus, cell cycle, replication

\section{Introduction}

Foot-and-mouth disease virus (FMDV), a member of the family Picornaviridae, genus Aphthovirus, is a small, nonenveloped RNA viruses containing a positive single strand RNA genome, which is the causative agent of foot-andmouth disease (FMD) characterized by the appearance of vesicles on the feet and in and around the mouth.

Studies on the cellular site of FMDV replication have shown that FMDV RNAs were predominantly localized in the basal cells of both mouth and foot epithelium during disease with visible cytopathology (unpublished data). During persistence, the viral RNA was localized in the basal cell layer of epithelia from the dorsal soft palate of animals not showing cytopathology [1]. Within a normal epithelium, the stratum basale cells are the cells involved in DNA synthesis and cell division. Some of the daughter cells of the stratum basale cells leave this cell cycle and

Received November 13, 2014; accepted December 7, 2014

Correspondence: zhangzhidong@caas.cn differentiate into the spinosum, granulosum and corneum cells. Previous studies on the reproduction of picornaviruses indicated the relationship of the kinetics of viral RNA synthesis in coordinated cells to the stage of the celldivision cycle [2-5]. For example, a link between the celldivision cycle and virus replication is seen with coxsackievirus B infection, where low-level of the virus reproduction occurred in quiescent cells but rapid viral protein expression was seen at the wound margin of the monolayer cells, resulting in the production of infectious virus. It appears that viral RNA synthesis is strongly related to the stages of the cell-division cycle, particularly at the later stages [2].

The cell-division cycle consists of four different phases: $\mathrm{G}_{0}$ phase is a resting phase where the cell has left the cycle and has stopped dividing; $G_{1}$ phase where cytoplasmic materials are accumulated; $S$ and $\mathrm{G}_{2}$ phases in where DNA replication occurs; and the $\mathrm{M}$ phase, where cell duplication occurs. It is unclear if FMDV synthesis in vivo is related to the cell-division cycle, but the restriction of FMDV to the certain cells of epithelia [6] suggests a possible link between FMDV replication in vivo and the status of cells at infection. To address this question, an in vitro epithelial cell model was developed to investigate if there was an association between the rate of virus replication and the type/status of infected epithelial cells. The results demonstrated that when cells were arrested at the $G_{1}$ or $G_{1} / S$ phase, high levels of viral RNA were detected by quantitative real-time reverse transcription PCR and extensive viral protein synthesis was observed by specific labeling techniques. When cells were arrested at the $\mathrm{G}_{2} / \mathrm{M}$ phase, however, reduced or no viral RNA synthesis was detected or viral protein synthesis observed.

\section{Materials and methods}

\subsection{Cells and virus}

Primary cultures of epithelial cells from tissues of ovine 
dorsal soft palate, tongue and kidney as well as bovine thyroid (BTY) were prepared according to standard procedures. Briefly, tissues were collected post mortem and transported in ice-cold Dulbecco's modified Eagle's medium (DMEM). After washing with phosphate buffered saline (PBS), the epithelial cell layer was separated from other contaminants, cut into $3-4 \mathrm{~mm}$ pieces and washed in $5 \mathrm{mmol} \cdot \mathrm{L}^{-1}$ DTT in ice-cold PBS. Tissues were then incubated overnight in protease $\left(0.4 \mathrm{mg} \cdot \mathrm{mL}^{-1}\right.$ in PBS $)$ at $4^{\circ} \mathrm{C}$. Protease was inactivated by fetal calf serum. Cells were pelleted and washed several times in DMEM before being filtered through a $100 \mu \mathrm{mol} \cdot \mathrm{L}^{-1}$ strainer followed by a $40 \mu \mathrm{mol} \cdot \mathrm{L}^{-1}$ filter. Cells were pelleted and re-suspended in DMEM/F12, supplemented with $5 \%$ fetal calf serum (FCS), and seeded at a concentration of $10^{5}$ cells per milliliter. Cultures were incubated at $37^{\circ} \mathrm{C}$, under $5 \% \mathrm{CO}_{2}$. Cell line ZZ-R127 (supplied by the Collection of Cell Lines in Veterinary Medicine of the Friedrich-LoefflerInstitute), was grown in DMEM and Ham's F12 (DMEM/ F12) supplemented 1:1 with 5\% FCS, at a concentration of $10^{5}$ cells per milliliter. Cells were incubated at $37^{\circ} \mathrm{C}$, under $5 \% \mathrm{CO}_{2}$.

\subsection{Virus isolation}

The infectivity of collected samples was assayed by inoculation of BTY cells [7].

\subsection{Quantitative real-time reverse transcription PCR (qRT-PCR)}

An automated extraction procedure was used for nucleic acid extraction of cell supernatants [8]. Quantitative PCR (qRT-PCR) were performed as previously described on a StrategeneMxPro 3005P QPCR System [9]. Results were analyzed by means of MxPro QPCR software and genome copy numbers per reaction were also calculated according to Quan et al. [10]. Statistical analyses were carried out by using a non-parametric test (independent samples $t$-test using Excel). $P<0.05$ was considered statistically significant.

\subsection{Cell wounding}

Monolayers of primary cells from ovine dorsal soft palate, tongue and kidney as well as BTY and ZZ-R127 monolayers were seeded onto glass coverslips and grown to $90 \%$ confluence. Monolayers were then wounded with a fine needle $2 \mathrm{~h}$ before inoculation with FMDV type OUKG34/2001 at multiplicity of infection of approximately 0.1 M.O.I. After incubation for $1 \mathrm{~h}$ at $37^{\circ} \mathrm{C}$ and $5 \%$ $\mathrm{CO}_{2}$, cells were washed three times with PBS and returned to an incubator. Cell supernatants were collected and glass coverslips were fixed at $0,4,8$ and $24 \mathrm{~h}$ post inoculation (hpi). Cell supernatants were stored at $-70^{\circ} \mathrm{C}$ for QPCR analyses and coverslips were fixed with $4 \%$ paraformalde- hyde for $15 \mathrm{~min}$ at room temperature and stored at $4{ }^{\circ} \mathrm{C}$ in $0.1 \%$ sodium azide (in PBS) for confocal laser scanning microscopy studies.

\subsection{Induction of cell quiescence}

Cellular quiescence was induced by serum depletion. A total of $10^{5}$ ZZ-R127 cells were seeded into each well of 24-well plates containing glass coverslips and were grown overnight in DMEM/F-12 medium (Invitrogen) plus 5\% FCS. To induce quiescence, the medium was then replaced with DMEM/F-12, without FCS, for $24 \mathrm{~h}$. Cells were then inoculated with FMDV and at 0,6 and 24 hpi cell supernatants were harvested and glass coverslips were fixed as described above.

\subsection{Cell cycle arrest}

Cell cycle inhibitors for each stage of the cell cycle were used in order to arrest the cycle at specific phases. Monolayers of ZZ-R127 cells were seeded into each well of 24-well plates containing glass coverslips and were grown overnight in DMEM/F-12 medium (Invitrogen) plus $5 \%$ FCS, at approximately $80 \%$ confluence. Cell cycle inhibitors: $\mathrm{G}_{1}$ inhibitor: hydroxyurea $\left(1 \mathrm{mmol} \cdot \mathrm{L}^{-1}\right)$, aphidicolin $\left(5 \mu \mathrm{g} \cdot \mathrm{mL}^{-1}\right) ; \mathrm{G}_{1} / \mathrm{S}$ inhibitors: mimosine $\left(300 \mu \mathrm{mol} \cdot \mathrm{L}^{-1}\right)$, rapamycin $\left(25 \mathrm{nmol} \cdot \mathrm{L}^{-1}\right) ; \mathrm{G}_{2} / \mathrm{M}$ inhibitors: nocodazole $\left(1 \mu \mathrm{g} \cdot \mathrm{mL}^{-1}\right)$ or taxol $\left(200 \mathrm{nmol} \cdot \mathrm{L}^{-1}\right)$, purchased from Sigma-Aldrich, were added to the cells for $16 \mathrm{~h}$, and incubated at $37^{\circ} \mathrm{C}, 5 \% \quad \mathrm{CO}_{2}$. Cells were inoculated with virus, as described above, and medium plus inhibitors, with or without fetal calf serum, was restored. After a $12 \mathrm{~h}$ incubation period at $37^{\circ} \mathrm{C}$ in $5 \%$ $\mathrm{CO}_{2}$, cell supernatants and glass coverslips were also processed as above.

\subsection{FMDV inactivation curve}

Taxol was tested for its capability to inactivate FMDV. Monolayers of ZZ-R127 cells were seeded into each well of 24-well plates containing glass coverslips and were grown in DMEM/F-12 medium (Invitrogen) plus 5\% FCS to $90 \%$ confluence. Virus was diluted (0.1 M.O.I.) in DMEM/F-12, both with or without taxol $\left(200 \mathrm{nmol} \cdot \mathrm{L}^{-1}\right)$. Virus dilutions were held at $22^{\circ} \mathrm{C}$, with gentle agitation. Cells were inoculated with 0.1 M.O.I. at 2,4 and $20 \mathrm{~h}$ post dilution (hpd) and incubated for $1 \mathrm{~h}$ at $37^{\circ} \mathrm{C}$ and $5 \% \mathrm{CO}_{2}$. Cells were washed three times with PBS, DMEM/F-12 medium plus 5\% FCS was replaced and cells were then returned to an incubator. Evidence of cytopathic effect (CPE) was observed for 3 days.

\subsection{Immunofluorescence microscopy}

Cell monolayers fixed on coverslips were permeabilized in $0.1 \%$ TritonX-100 (in PBS) for $15 \mathrm{~min}$, at room tempera- 
ture, on a shaker and blocked for 30 min with $0.5 \%$ bovine serum albumin (BSA) in PBS. Cells were washed three times with PBS and then incubated with pre-diluted primary antibodies for $1 \mathrm{~h}$ at room temperature, on a shaker. Cells were washed three times with PBS and then incubated with secondary, isotype specific antibodies conjugated to Alexa dyes (Molecular Probes) for $1 \mathrm{~h}$ at room temperature, on a shaker. Cells were washed as before and incubated for $10 \mathrm{~min}$ with 4,6-diamino-2phenylindole (DAPI; Molecular Probes). Cells were then washed with deionised water and mounted onto glass slides using Vectashield Mounting Medium (Vector Laboratories). Slides were observed with a Leica TCS/ SP2 confocal microscope.

All primary antibodies were diluted in $0.5 \%$ BSA in PBS. IB11, mouse monoclonal, raised against $146 \mathrm{~S}$ FMDV type $\mathrm{O} 1$ antigen, specific for conformational, non-neutralising epitopes of the FMDV capsid, diluted at 1:10, kindly provided by Nicholas Jullef, Immunology Department, Pirbright Institute. Ki67 (mouse anti-human IgG1, clone M1-B1, Dako) diluted at 1:100. Zo-1 (antimouse IgG1, Invitrogen) diluted 1:50. $\alpha \mathrm{v} \beta 6$ (mouse anti human integrin, $\operatorname{IgG} 2 \mathrm{a}$, Chemicom) and $\beta 1$ (mouse antihuman IgG1, Chemicon) both diluted at 1:100. Cytokeratin (pan anti-mouse IgG1, Sigma) diluted at 1:100. Secondary antibodies were diluted in $0.5 \%$ BSA (in PBS), at 1:200. Goat anti-mouse IgG2a or IgG2b 488 and goat anti-mouse IgG1 568 were obtained from Alexa Fluor (Molecular Probes).

\section{Results}

3.1 No correlation of the quiescent status of the cells $\left(\mathrm{G}_{0}\right.$ phase) with FMDV RNA synthesis

The quiescent ZZ-R 127 cells were generated by starvation of FCS (Quiesc) $24 \mathrm{~h}$ before inoculation with FMDV. Some cells (Quiesc/continual $\mathrm{G}_{0}$ cells) remained at $\mathrm{G}_{0}$ phase by having no addition of FCS to their medium throughout the experiment, whereas others (Quiesc/cycling cells) were allowed to cycle by the addition of FCS after $24 \mathrm{~h}$ starvation and virus inoculation. The virus replication in the quiescent cells at 0,6 and 24 hpi was assessed by qRT-PCR.

As shown in Fig. 1, FMDV replication occurred in the quiescent cells. At 6 and 24 hpi, there was no difference in the amount of viral genomes in cells between cells that were starved prior to inoculation (Quiesc/continual $\mathrm{G}_{0}$ cells; 5.4 and $9.1 \log _{10}$ copies per milliliter at 6 and $24 \mathrm{hpi}$, respectively) and control cells that were not starved (No Quiesc/cycling cells, 6.4 and $9.2 \log _{10}$ copies per milliliter at 6 and 24 hpi, respectively). Further study showed that release of the cells (starved cells before virus inoculation) from $\mathrm{G}_{0}$ phase by the addition of serum in the media after

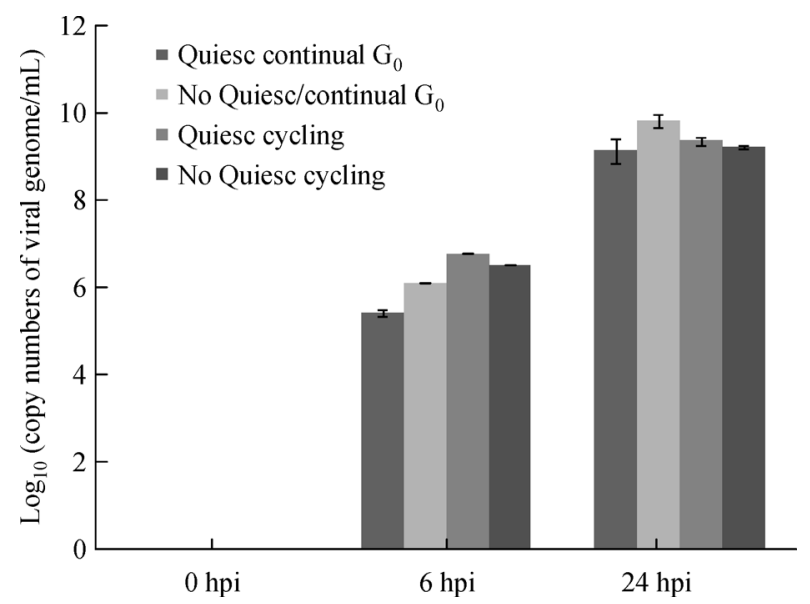

Fig. 1 Virus replication in quiescent cells $\left(\mathrm{G}_{0}\right)$. The graph shows results obtained with ZZ-R127 monolayers that were either starved of FCS (Quiesc) or not (No Quiesc) $24 \mathrm{~h}$ before virus inoculation. Some monolayers remained at 'continual $\mathrm{G}_{0}$ ', i.e., no FCS was added to their medium throughout the experiment, whereas others were allowed to cycle (cycling), by the addition of FCS after $24 \mathrm{~h}$ starvation and virus inoculation. Error bars represent standard deviation obtained with average of qRT-PCR expressed as $\log _{10}$ (copy numbers of viral genome per milliliter). Samples were collected and analyzed at 0,6 and $24 \mathrm{~h}$ post-inoculation (hpi).

inoculation with FMDV had no impact on FMDV RNA synthesis, even though a higher level of viral RNA was detected in these Quiesc/cycling cells [6.7 $\log _{10}$ (copies per milliliter)] at 6 hpi compared to those at Quiesc/Continual $\mathrm{G}_{0}\left[5.4 \log _{10}\right.$ (copies per milliliter) $\left.(P=0.11, P>0.005)\right]$. Similarly, the viral RNA level in Quiesc/continual $\mathrm{G}_{0}$ cells was higher than in cells that were starved after inoculation [No Quiesc/continual $\mathrm{G}_{0} ; 6.1$ and $9.8 \log _{10}$ (copies per milliliter) at 6 and $24 \mathrm{hpi}$, respectively)]. Qualitative immunofluorescence supported the data obtained with qRT-PCR (data not shown). These results indicated that FMDV RNA synthesis occurs in quiescent cells $\left(\mathrm{G}_{0}\right.$ phase).

3.2 Viral replication does not preferentially occur in proliferating cells

It has been suggested that proliferating cells near the wounded area preferentially support Coxsackievirus protein production [11]. Therefore wounding the cell monolayer to induce proliferating cells was carried out to investigate if FMDV replication preferentially occur in proliferating cells. The level of viral RNA synthesis was analyzed by qRT-PCR. As shown in Fig. 2, the difference in number of viral genome copies between injured and uninjured monolayers was negligible for the majority of samples analyzed (Fig. 2). Confocal microscopy also showed that there was no trend in relation to the labeling of viral protein when comparing injured with uninjured 
monolayers (data not shown). The pattern of labeling observed was not restricted to and it was no more intense at or near the injury site as has been reported for other viruses $[11,12]$. These observations agree with findings of other FMDV workers [13], indicating proliferating cells do not preferentially support viral replication.

3.3 The stages of the cell-division cycle influence viral propagation

To investigate the impact of cell cycle status on viral RNA synthesis, cell cycle inhibitors were used to arrest cells at

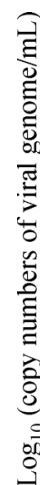
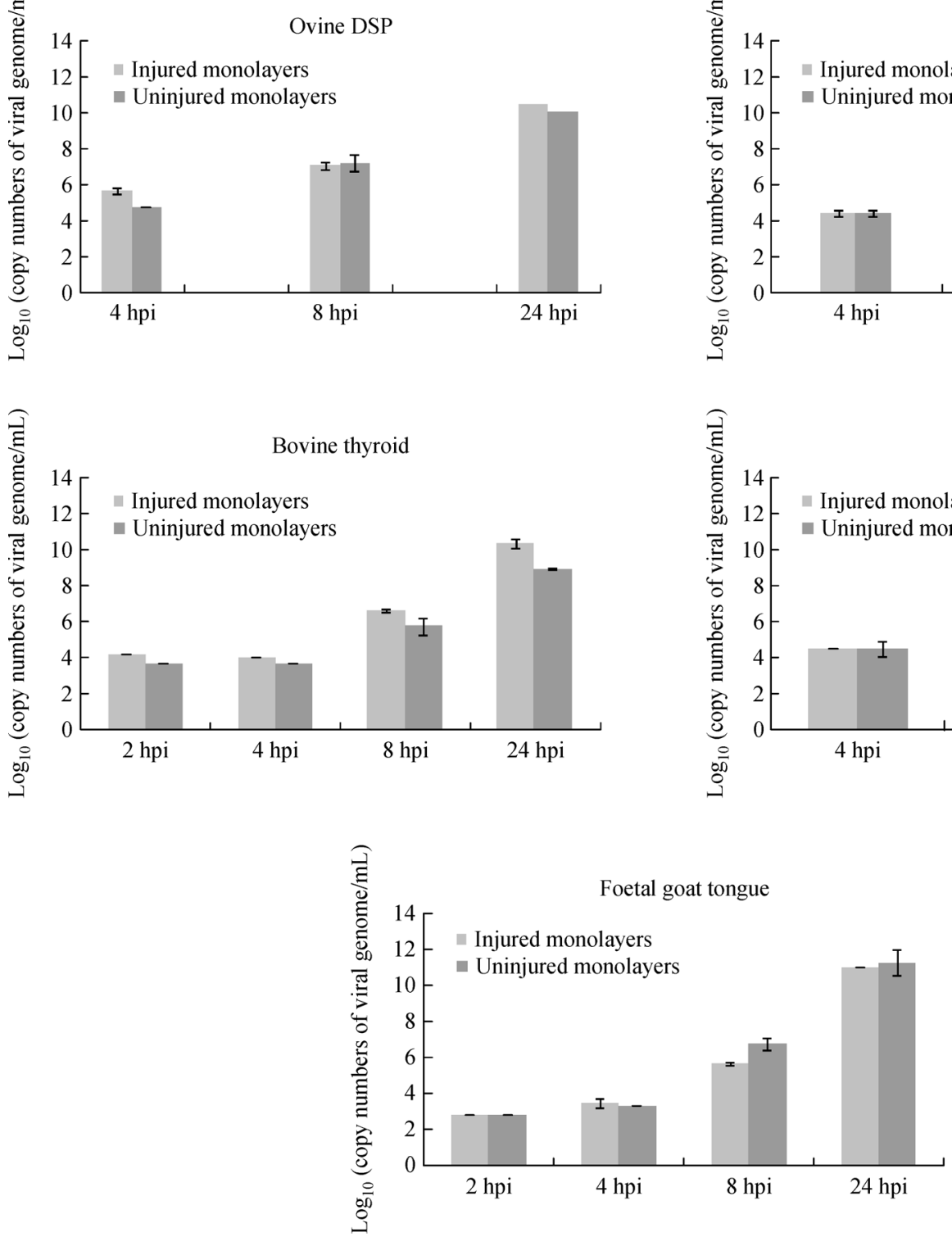

Fig. 2 Virus replication in proliferating cells. Graphs show comparisons of qRT-PCR results expressed as $\log _{10}$ (copy numbers of viral genome per milliliter), from injured and uninjured monolayers of several primary epithelial cell cultures and the epithelial cell line ZZR127 (fetal goat tongue). Samples were collected and analyzed at 2 (bovine thyroid and fetal goat tongue only), 4,8 and $24 \mathrm{~h}$ postinoculation (hpi). different stages and then infected with FMDV. As shown in Fig. 3, when the ZZ-R127 cells were blocked at $\mathrm{G}_{1}$ phase with inhibitors mimosine or rapamycin with or without $5 \%$ FCS in medium, the level of viral RNA synthesis at $12 \mathrm{hpi}$ was not significantly different from that in the control cells. Similarly, when the cells were arrested at $\mathrm{G}_{1} / \mathrm{S}$ phase using hydroxyurea as inhibitor, there was no significant difference in the level of viral RNA synthesis at $12 \mathrm{hpi}$ from those control cells. However, when the cells were blocked at $\mathrm{G}_{2} / \mathrm{M}$ phase with the inhibitor taxol with or without FCS (data not shown) in the medium, no viral RNA was detected, which was significantly different from
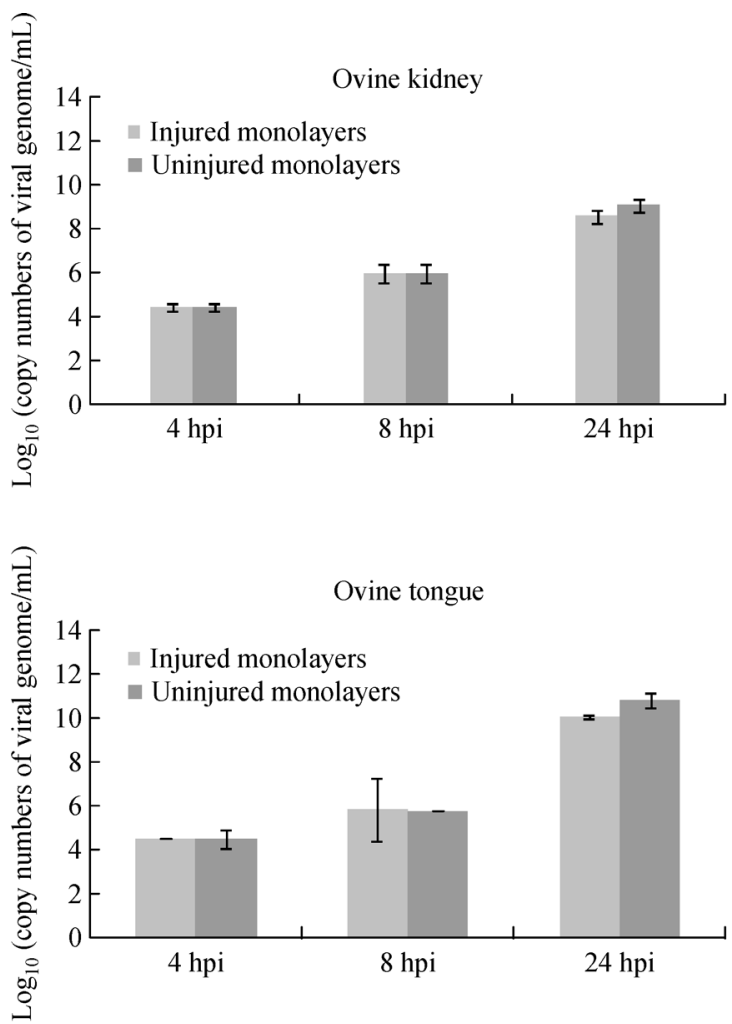


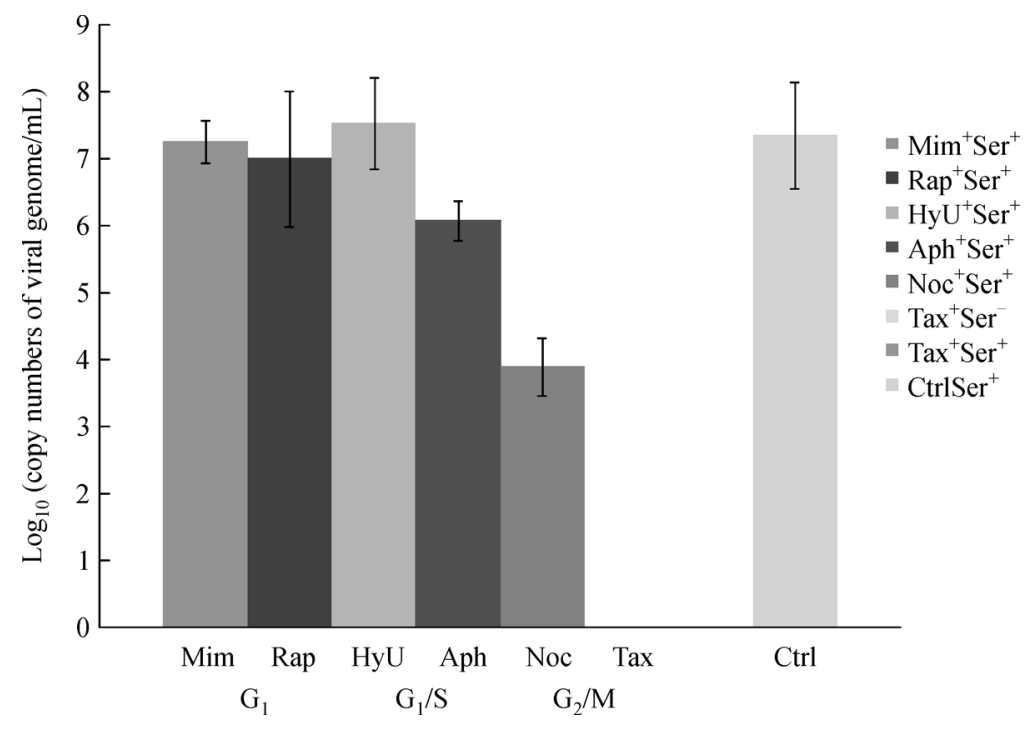

Fig. 3 Virus replication during cell cycle arrest. $\log _{10}$ (FMDV genome copy numbers per milliliter) detected from different cells arrested at $\mathrm{G}_{1}, \mathrm{G}_{1} / \mathrm{S}$ or $\mathrm{G}_{2} / \mathrm{M}$ phase of the cell cycle after a $12 \mathrm{~h}$ exposure to FMDV. Cells were cultured in medium containing $5 \%$ (Ser ${ }^{+}$) of fetal calf serum. Inhibitors used were mimosine (Mim), rapamycin (Rap), hydroxyurea (HyU), aphidicolin (Aph), nocodazole (Noc), taxol (Tax) and blank control (Ctrl). Error bars represent standard deviation of average of QPCR results.

those control cells. Similarly, the level of viral RNA synthesis at $12 \mathrm{hpi}$ [4 $\log$ (copies per milliliter)] in the cells treated with another inhibitor Nocodazol with CFS in medium was considerably lower than those control cells [7.4 $\log _{10}$ (copies per milliliter); $P=0.018, P<0.05$ ]. To test if taxol has a direct inhibitory effect on viral RNA synthesis, an experiment where cells were inoculated with FMDV and exposed to taxol for a period up to $72 \mathrm{~h}$ was conducted. The results showed virus genome was detected and viral protein was observed at $18 \mathrm{hpi}$ and the difference between samples treated with and without the taxol [9.7 and $9.8 \log _{10}$ (copies per milliliter), respectively] was negligible (Fig. 4). Further study showed that taxol was unable to inactive FMDV (data not shown). The result indicated that there is an association of FMDV RNA synthesis with the stages of the cell-division cycle.

Ki67 (a nuclear protein) is expressed in proliferating cells during late $G_{1}, S, G_{2}$ and $M$ phases but not in resting cells in the $\mathrm{G}_{0}$ phase of the cell-division cycle. To perform an assessment of the influence of these cell cycle inhibitors on cell proliferating function, the ZZ-R127 cells treated with the inhibitors was stained with Ki67 antibody. Quantification of the proliferation rate can then be determined by the Ki67 index which is the number of

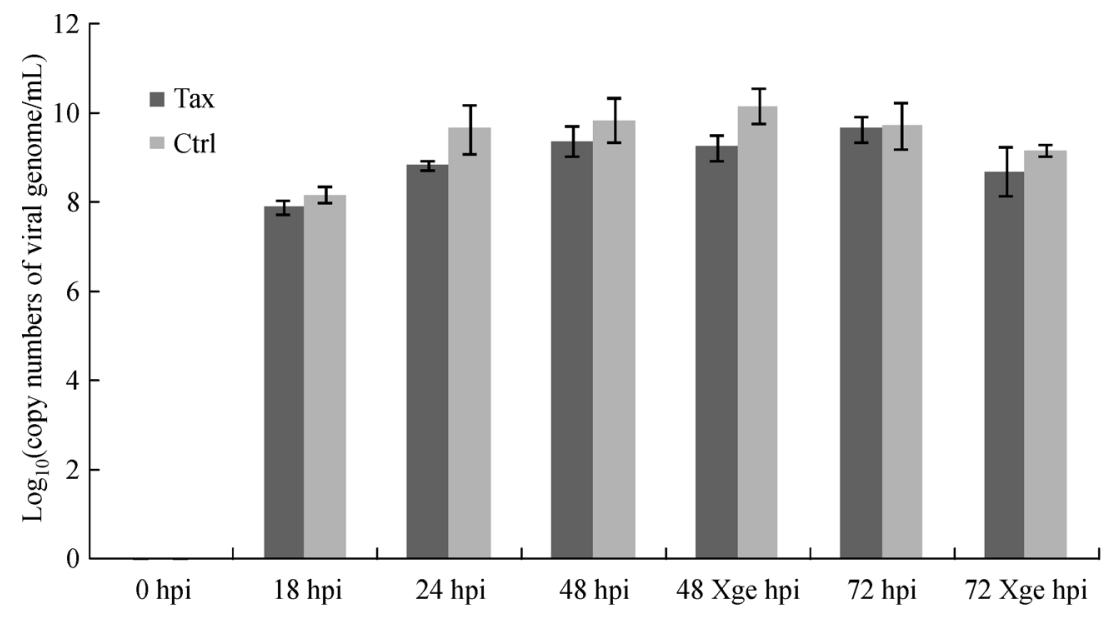

Fig. 4 Comparison of virus kinetics between cultures of medium containing taxol and cultures without this inhibitor. Taxol (Tax) was added to monolayers $24 \mathrm{~h}$ before virus inoculation; control monolayers (Ctrl) were maintained inhibitor-free. Samples were collected at $18,24,48$ and $72 \mathrm{~h}$ post inoculation. Taxol half-life was assessed by replacing medium at 48 and $72 \mathrm{~h}$ post-inoculation ( $48 \mathrm{Xge}$ and 72 Xge, respectively) and comparing with those samples where medium was not replaced. 
Ki67 positive cells among the total number of resting cells (Fig. 5). Confocal microscopy studies (Fig. 6) showed that the highest number of Ki67 labeled nuclei was observed in FMDV inoculated monolayers which were arrested with nocodazole (plus FCS) or hydroxyurea (without FCS). However, there was no relationship between FMDV RNA synthesis (Fig. 3) and the number of proliferating cells (Figs. 5 and 6) which further indicates that viral replication do not preferentially occur in proliferating cells.

\section{Discussion}

Replication of FMDV in cells in vitro in quiescent cells, five different types of proliferating cells and cells arrested at different phases of the cell division cycle was investigated in this study. The results suggest that when cells are arrested at the $G_{2} / M$ phase, reduced or no viral RNA synthesis was detected, indicating a possible link between FMDV replication and the cell-division cycle status.

Our studies have demonstrated that, when cells were first synchronized at $\mathrm{G}_{2} / \mathrm{M}$ phase with Nocodazole and then inoculated with FMDV, the amount of viral genome detected decreased greatly or was not detected at all (taxol). Cells synchronized at either $\mathrm{G}_{1} / \mathrm{S}$ or $\mathrm{G}_{1}$ phase showed a high number of viral genome copies, similar to those observed in control samples, i.e., where cells were not exposed to inhibitors. This result corroborates previous findings which suggest that certain viral and cellular IRES elements are active during the $\mathrm{G}_{2} / \mathrm{M}$ phase of the cell cycle. The protein synthesis of positive-strand RNA viruses is under the control of a defined IRES. There might be an advantage for a virus to arrest cells in the $\mathrm{G}_{2} / \mathrm{M}$ stage of the cell-division cycle because at these stages IRES-dependent translation of some (but not all) cellular and certain viral mRNAs appears to be optimal in comparison to other stages of the cell-division cycle [14]. Furthermore, the eIF4E subunit of eIF4F is dephosphorylated at the $\mathrm{G}_{2} / \mathrm{M}$ stage, and this is correlated with diminished cap-dependent translation [9]. On the other hand, cells forced into $\mathrm{G}_{0}$, by either serum starvation or wounding of monolayers, did not show an increase in copy numbers of viral genome, as observed with other Picornaviruses [12] in which viral replication does not preferentially occur in proliferating cells.

Taxol gave rise to the most intriguing data of the two $\mathrm{G}_{2} / \mathrm{M}$ inhibitors chosen for the experiments. Taxol is a plant alkaloid commonly used to perturb microtubule dynamics [15]. As a part of the cell's apparatus, microtubules are essential for cell division and replication, hence inhibition of these structures will lead to cell death. It has been demonstrated that taxol inhibits HeLa cell proliferation by inducing a sustained mitotic block at the metaphase/anaphase boundary [16]. However, our results suggest that taxol did not inhibit cell proliferation of both normal or FMDV inoculated ZZ-R127 cells. We have also demonstrated that taxol did not inactivate FMDV. However, there seemed to be a delay on the development of CPE of taxol diluted samples which remains to be explained. One hypothesis is that a taxol induced modification of UTRs, located at each end of the viral RNA, could impact on the control of gene expression and viral replication and, consequently, on inefficient viral replication. Nevertheless, further studies are required to understand the effects of taxol on viral-specific RNA
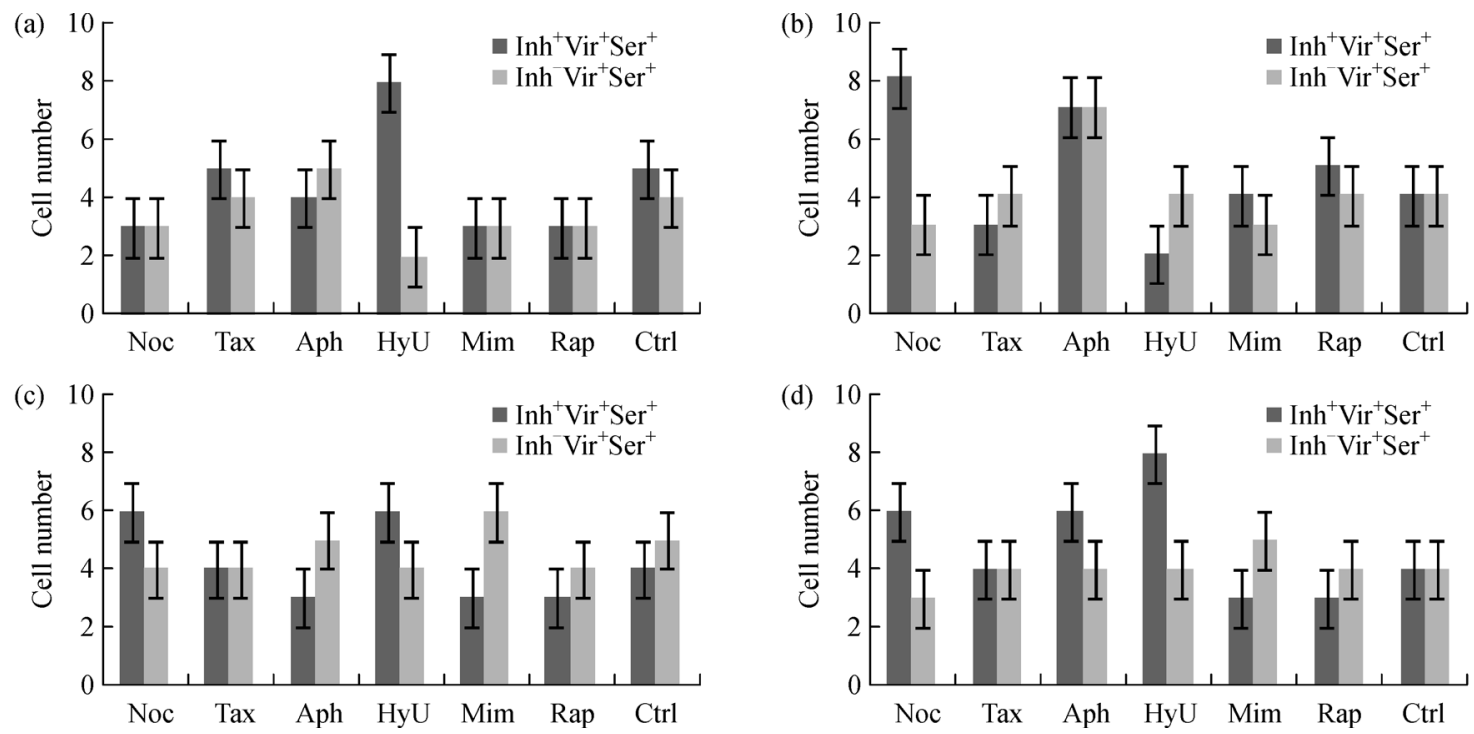

Fig. 5 Cell proliferation status was determined by the Ki67 index which is the number of Ki67 positive cells among the total number of resting cells. (a) FMDV inoculated monolayers with fetal calf serum (FCS); (b) FMDV inoculated monolayers without FCS; (c) virus control, i.e., non-inoculated monolayers, with FCS; (d) virus control, i.e., non-inoculated monolayers, without FCS. Inhibitors used were nocodazole (Noc), taxol (Tax), aphidicolin (Aph), hydroxyurea (HyU), mimosine (Mim), rapamycin (Rap) and blank control (Ctrl). 


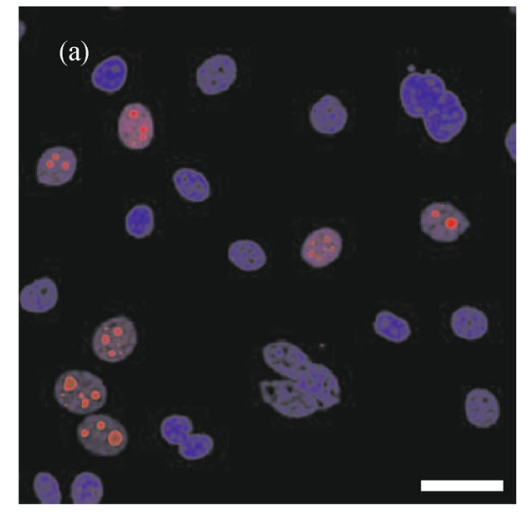

Aphidicolin

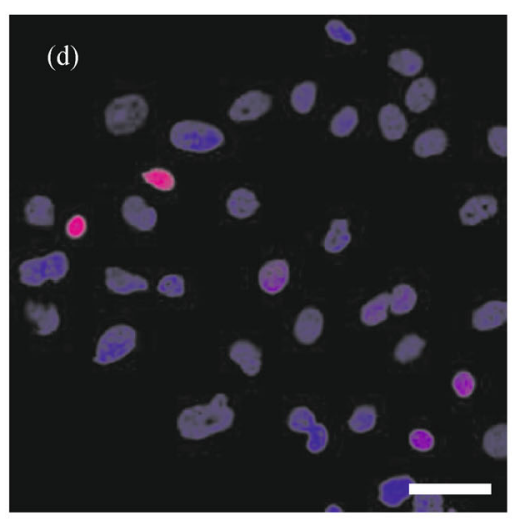

Rapamycin

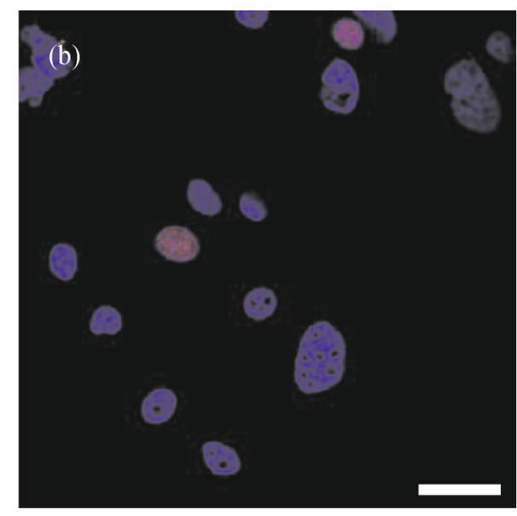

Hydroxyurea

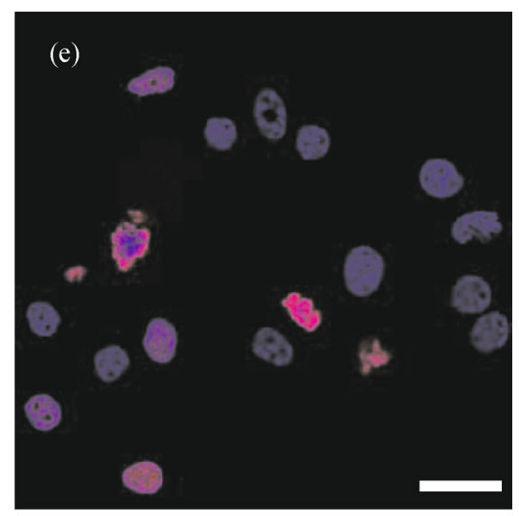

Nocodazole

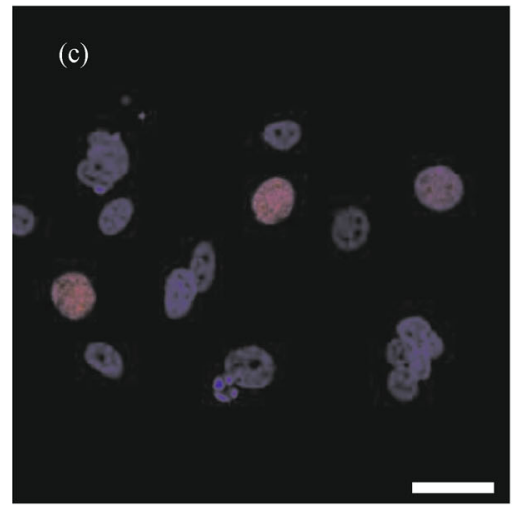

Mimosine

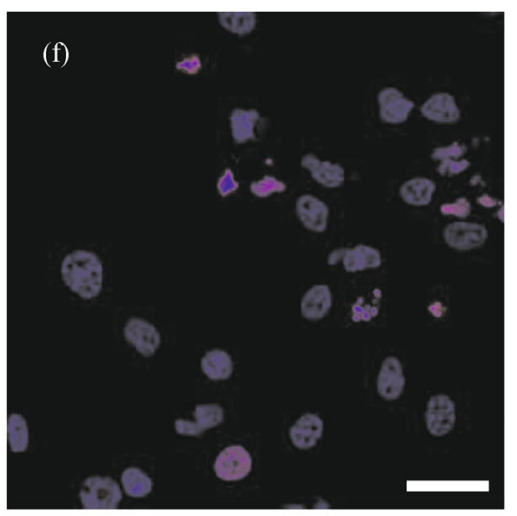

Taxol

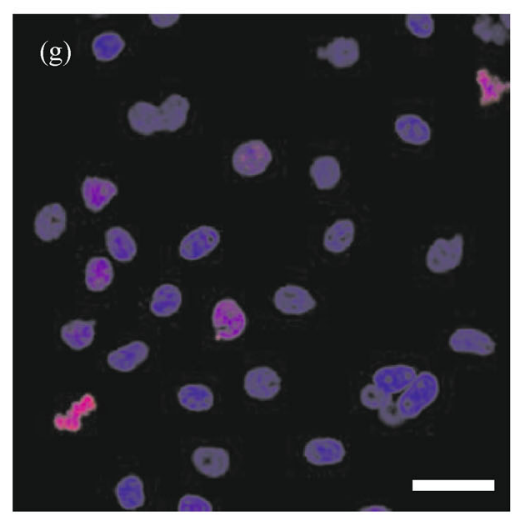

Control

Fig. 6 ZZ-R127 cell proliferation profile. Ki67 antibody was used for the assessment of the proliferation rate of cells exposed to cell cycle inhibitors. Cell cycle was arrested at $\mathrm{G}_{1}$ with inhibitors Aphidicolin (a) and/or Hydroxyurea (b), at $\mathrm{G}_{1} / \mathrm{S}$ with inhibitors Mimosine (c) and/or Rapamycin (d) and at $\mathrm{G}_{2} / \mathrm{M}$ with inhibitors Nocodozole (e) and/or Taxol (f). Cell monolayer control is represented in Control (g). Cell monolayers were fixed $16 \mathrm{~h}$ later and labeled with primary antibody Ki67 and goat anti-mouse AlexaFluor 568. Nuclei were stained in blue with DAPI. Scale bar: $40 \mu \mathrm{m}$.

structures which work in close association with host and viral proteins.

\section{Conclusions}

Reduced or no viral RNA synthesis was detected in cells arrested at the $\mathrm{G}_{2} / \mathrm{M}$ phase, indicating there is a possible link between FMDV replication and the cell-division cycle status.

Acknowledgements This work was mainly supported by the Biotechnology and Biological Sciences Research Council (China partnership award and project BB/E003435/1), UK. 
Compliance with ethics guidelines Claudia Doel, Zhidong Zhang, Lise Mazelet, Ryan Waters and John Bashiruddin declare that they have no conflict of interest or financial conflicts to disclose.

All applicable institutional and national guidelines for the care and use of animals were followed.

\section{References}

1. Zhang Z D, Kitching R P. The localization of persistent foot and mouth disease virus in the epithelial cells of the soft palate and pharynx. Journal of Comparative Pathology, 2001, 124(2-3): 8994

2. Eremenko T, Benedetto A, Volpe P. Virus infection as a function of the host cell life cycle: replication of poliovirus RNA. Journal of General Virology, 1972, 16(1): 61-68

3. Lake R S, Winkler D C, Ludwig E H. Delay of mengovirus-induced cytopathology in mitotic L-cells. Journal of Virology, 1970, 5(2): 262-263

4. Mallucci L, Wells V, Beare D. Cell cycle position and expression of encephalomyocarditis virus in mouse embryo fibroblasts. Journal of General Virology, 1985, 66(7): 1501-1506

5. Suarez M, Contreras G, Fridlender B. Multiplication of Coxsackie B1 virus in synchronized HeLa cells. Journal of Virology, 1975, 16 (5): 1337-1339

6. Zhang Z D, Hutchings G, Kitching P, Alexandersen S. Interferongamma cures cells persistently infected with foot-and-mouth disease virus. Archives of Virology, 2002, 147: 2157-2167

7. Snowdon W A. Growth of foot-and mouth disease virus in monolayer cultures of calf thyroid cells. Nature, 1966, 210(5040): 1079-1080
8. Reid S M, Grierson S S, Ferris N P, Hutchings G H, Alexandersen S. Evaluation of automated RT-PCR to accelerate the laboratory diagnosis of foot-and-mouth disease virus. Journal of Virological Methods, 2003, 107(2): 129-139

9. Huang J T, Schneider R J. Adenovirus inhibition of cellular protein synthesis involves inactivation of cap-binding protein. Cell, 1991, 65(2): 271-280

10. Quan M, Murphy C M, Zhang Z P, Alexandersen S. Determinants of early foot-and-mouth disease virus dynamics in pigs. Journal of Comparative Pathology, 2004, 131(4): 294-307

11. Feuer R, Mena I, Pagarigan R, Slifka M K, Whitton J L. Cell cycle status affects coxsackievirus replication, persistence, and reactivation in vitro. Journal of Virology, 2002, 76(9): 4430-4440

12. Feuer R, Mena I, Pagarigan R R, Hassett D E, Whitton J L. Coxsackievirus replication and the cell cycle: a potential regulatory mechanism for viral persistence/latency. Medical Microbiology and Immunology, 2004, 193(2-3): 83-90

13. Martín-Acebes M A, Herrera M, Armas-Portela R, Domingo E, Sobrino F. Cell density-dependent expression of viral antigens during persistence of foot-and-mouth disease virus in cell culture. Virology, 2010, 403(1): 47-55

14. Dove B, Brooks G, Bicknell K, Wurm T, Hiscox J A. Cell cycle perturbations induced by infection with the coronavirus infectious bronchitis virus and their effect on virus replication. Journal of Virology, 2006, 80(8): 4147-4156

15. Schiff P B, Fant J, Horwitz S B. Promotion of microtubule assembly in vitro by taxol. Nature, 1979, 277(5698): 665-667

16. Jordan M A, Toso R J, Thrower D, Wilson L. Mechanism of mitotic block and inhibition of cell proliferation by taxol at low concentrations. Proceedings of the National Academy of Sciences of the United States of America, 1993, 90(20): 9552-9556 